\title{
Extremely large magnetoresistance and ultrahigh mobility in the topological Weyl semimetal candidate $\mathrm{NbP}$
}

\author{
Chandra Shekhar ${ }^{1}$, Ajaya K. Nayak ${ }^{1,2}$, Yan Sun ${ }^{1}$, Marcus Schmidtt, Michael Nicklas ${ }^{1}$, Inge Leermakers ${ }^{3}$, \\ Uli Zeitler ${ }^{3}$, Yurii Skourski ${ }^{4}$, Jochen Wosnitza ${ }^{4}$, Zhongkai Liu' ${ }^{5}$, Yulin Chen ${ }^{6}$, Walter Schnelle', \\ Horst Borrmann ${ }^{1}$, Yuri Grin', Claudia Felser ${ }^{1}$ and Binghai Yan ${ }^{1,7 \star}$
}

Recent experiments have revealed spectacular transport properties in semimetals, such as the large, non-saturating magne-

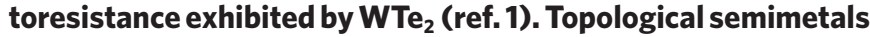
with massless relativistic electrons have also been predicted ${ }^{2}$ as three-dimensional analogues of graphene ${ }^{3}$. These systems are known as Weyl semimetals, and are predicted to have a range of exotic transport properties and surface states ${ }^{4-7}$, distinct from those of topological insulators ${ }^{8,9}$. Here we examine the magneto-transport properties of $\mathrm{NbP}$, a material the band structure of which has been predicted to combine the hallmarks of a Weyl semimetal ${ }^{10,11}$ with those of a normal semimetal. We observe an extremely large magnetoresistance of $850,000 \%$ at $1.85 \mathrm{~K}(250 \%$ at room temperature $)$ in a magnetic field of up to $9 \mathrm{~T}$, without any signs of saturation, and an ultrahigh carrier mobility of $5 \times 10^{6} \mathrm{~cm}^{2} \mathrm{~V}^{-1} \mathrm{~s}^{-1}$ that accompanied by strong Shubnikov-de Haas (SdH) oscillations. NbP therefore presents a unique example of a material combining topological and conventional electronic phases, with intriguing physical properties resulting from their interplay.

A Weyl semimetal (WSM) is a three-dimensional analogue of graphene, in which the conduction and valence bands cross near the Fermi energy. The band-crossing point, the so-called Weyl point, acts as a magnetic monopole (a singular point of Berry curvature) in momentum space and always comes in a pairs. Unusual transport properties and surface states such as Fermi arcs are predicted, stimulating strong interest in realizing the WSM state in real materials $^{2,12,13}$. If the time-reversal and inversion symmetries are respected, a pair of Weyl points can become degenerate in energy as a result of the crystal symmetry, forming another topological phase called a Dirac semimetal ${ }^{14,15}$. WSMs and Dirac semimetals usually exhibit very high mobilities, possibly attributed to the high Fermi velocity of massless Dirac states, as observed in transport experiments (such as $\mathrm{Cd}_{3} \mathrm{As}_{2}$; refs 16,17). Generally, semimetals are new platforms to realize a huge magnetoresistance (MR; refs 18,19), an effect that has been pursued intensively in emerging materials in recent years, because of its significant application in state-of-theart information technologies ${ }^{20}$. Electrical transport in a semimetal usually consists of two types of carriers (electrons and holes), leading to a large MR when a magnetic field is applied at an electron-hole resonance $^{1,21}$. In a simple Hall effect set-up, the transverse current

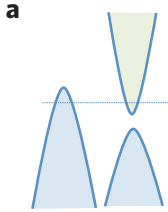

$\mathrm{Bi}, \mathrm{WTe}_{2}$ b

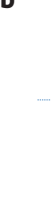
Ag-chalcogenides,
Heuslers c

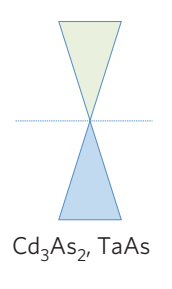

d

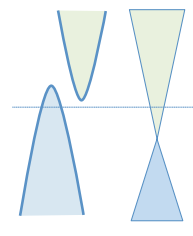

$\mathrm{NbP}$
Figure 1 | Band structure for different semimetals. Schematic illustration of different types of semimetals and representative materials. a, Normal semimetal with the coexistence of electron and hole pockets. $\mathbf{b}$, Semimetal with quadratic conduction and valence bands touching at the same momentum point. c, Weyl semimetal and Dirac semimetal. d, Semimetal with one hole pocket from the normal quadratic band and one electron pocket from the linear Weyl semimetal band for NbP. The valence and conduction bands are indicated by blue and green shading, respectively. The Fermi energy is marked in each case by a horizontal line.

carried by a particular type of carrier may be non-zero, although no net transverse current flows when the currents carried by the electrons and holes compensate for each other. These nonzero transverse currents will experience a Lorentz force caused by the magnetic field in the inverse-longitudinal direction. Such a back flow of carriers eventually increases the apparent longitudinal resistance, resulting in an extremely high MR that is much stronger than that in normal metals and semiconductors. Thus, it is crucial to obtain high-purity samples to realize a balance between electrons and holes and a high carrier mobility $(\mu)$ as well, both of which will enhance the MR effect.

Elemental Bi (refs 22-25) and $\mathrm{WTe}_{2}$ (ref. 1) exhibit a high MR as typical examples of semimetals, in which electron and hole pockets coexist on the Fermi surface (Fig. 1a). There is a special type of semimetal whose conduction-band bottom and valenceband top touch the Fermi surface at the same point in momentum (k) space (Fig. 1b,c). Many such semimetals exhibit a high carrier mobility and relatively large MR, with a linear dependence on the magnetic field, such as zero-gap topological-insulator silver chalcogenides ${ }^{26,27}$ and Heusler compound ${ }^{28-30}$, the Dirac semimetal $\mathrm{Cd}_{3} \mathrm{As}_{2}\left(\mu=9 \times 10^{6} \mathrm{~cm}^{2} \mathrm{~V}^{-1} \mathrm{~s}^{-1}\right.$ at $5 \mathrm{~K}, \mathrm{MR}=1,500 \%$ at $1.5 \mathrm{~K}$ and

${ }^{1}$ Max Planck Institute for Chemical Physics of Solids, 01187 Dresden, Germany. ${ }^{2}$ Max Planck Institute of Microstructure Physics, Weinberg 2, D-06120 Halle, Germany. ${ }^{3}$ High Field Magnet Laboratory (HFML-EMFL), Radboud University, Toernooiveld 7, 6525 ED Nijmegen, The Netherlands. ${ }^{4}$ Dresden High Magnetic Field Laboratory (HLD-EMFL), Helmholtz-Zentrum Dresden-Rossendorf, 01328 Dresden, Germany. ${ }^{5}$ Diamond Light Source, Harwell Science and Innovation Campus, Fermi Avenue, Didcot, Oxfordshire OX11 OQX, UK. ${ }^{6}$ Physics Department, Oxford University, Oxford OX1 3PU, UK. ${ }^{7}$ Max Planck Institute for the Physics of Complex Systems, 01187 Dresden, Germany. *e-mail: yan@cpfs.mpg.de 
a

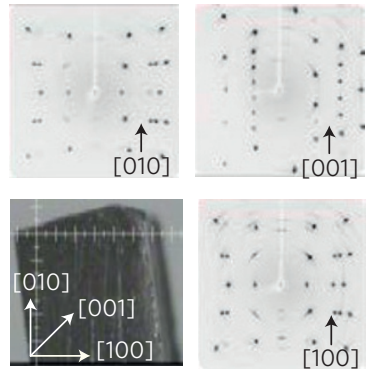

b

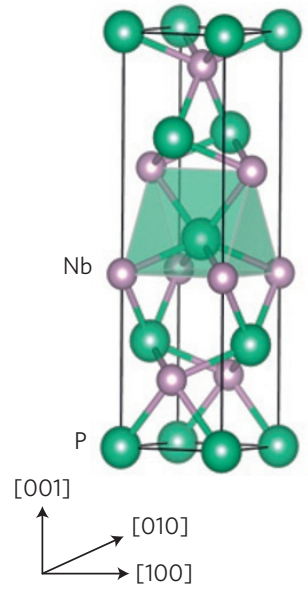

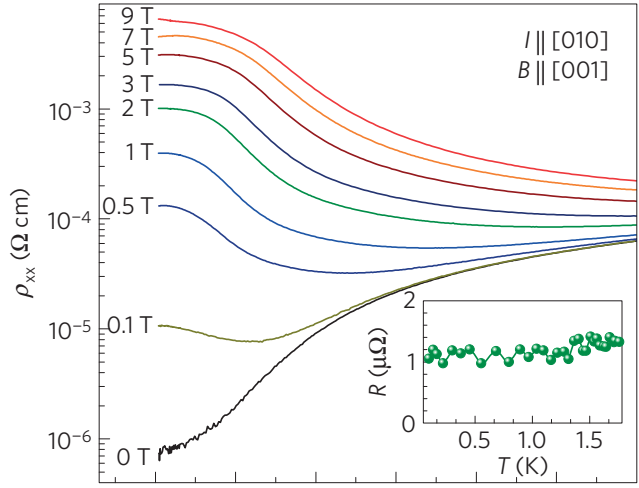

e

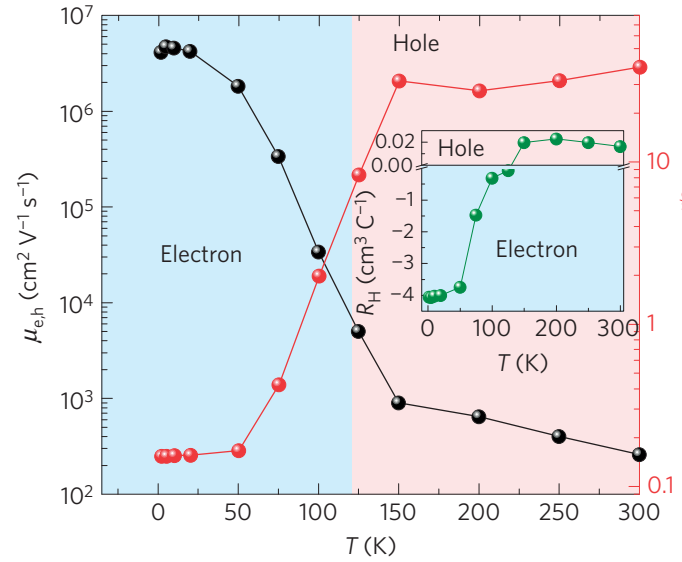

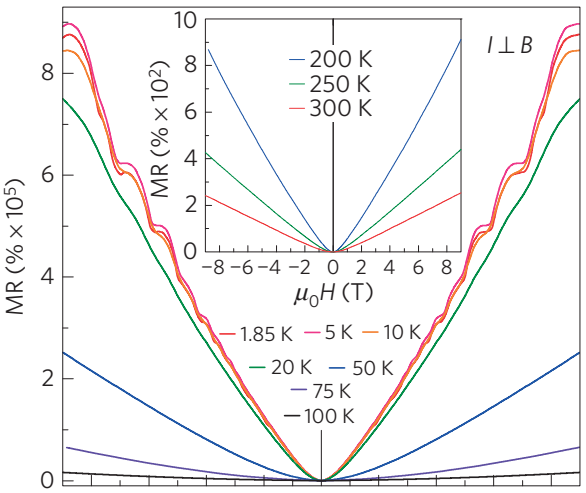

f

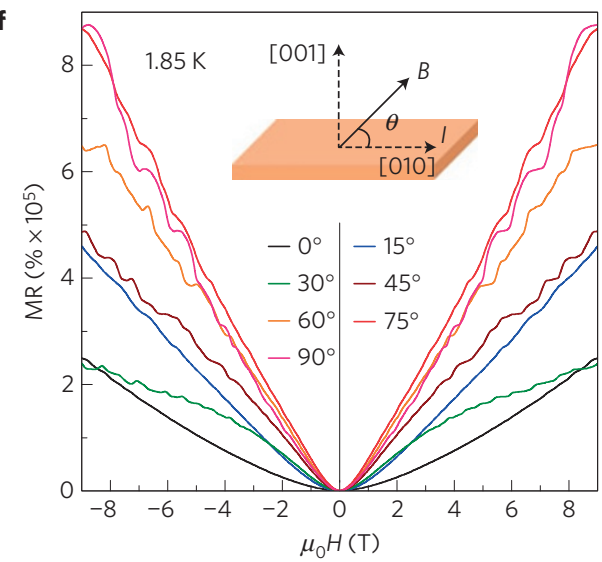

Figure $\mathbf{2}$ | Crystal structure, magnetoresistance and mobility. a, Orientation of the measured single-crystal NbP with the respective $\mathrm{X}$-ray diffraction axial oscillation patterns. $\mathbf{b}$, Crystal structure of $\mathrm{NbP}$ in a body-centred-tetragonal lattice. $\mathbf{c}$, Temperature dependence of the resistivity, $\rho_{x x}$, measured at different transverse magnetic fields, as labelled next to the corresponding curve. The inset of $\mathbf{c}$ shows the temperature dependence of resistance measured in zero field. d, Transverse magnetoresistance measured at different temperatures in magnetic fields up to $9 \mathrm{~T}$. The inset shows the magnetoresistance at higher temperatures. e, Temperature dependence of the mobility (left ordinate) and the carrier density (right ordinate). The inset shows the evolution of the Hall coefficient with temperature. The temperature regimes where electrons and holes act as the main charge carriers are marked with blue and red shading, respectively. $\mathbf{f}$, Magnetoresistance measured at different angles, $\theta$, between the current $(I)$ and the magnetic field $(B)$ as shown schematically in the inset.

14.5 T; refs 16,31-33), and the WSM TaAs $\left(\mu=5 \times 10^{5} \mathrm{~cm}^{2} \mathrm{~V}^{-1} \mathrm{~s}^{-1}\right.$ at $2 \mathrm{~K}, \mathrm{MR}=5.4 \times 10^{5} \%$ at $10 \mathrm{~K}$ and $9 \mathrm{~T}$; ref. 34$)$. The high mobility may originate from the linear or nearly linear energy dispersion. The unsaturated linear MR is interpreted as a classical effect due to the strong inhomogeneity in the carrier density ${ }^{35}$ or as a quantum effect due to the linear energy dispersion at the band touching point ${ }^{36}$. The semimetal NbP combines the main features of the $\mathrm{WTe}_{2}$-type (showing extremely large MR) and $\mathrm{Cd}_{3} \mathrm{As}_{2}$-type (showing ultrahigh mobility) semimetals in the band structure (Fig. 1d), exhibiting hole pockets from normal quadratic bands and electron pockets from linear Weyl bands. As we will see, NbP exhibits an ultrahigh carrier mobility, comparable to that of $\mathrm{Cd}_{3} \mathrm{As}_{2}$, and an extremely large $\mathrm{MR}$, surpassing that of $\mathrm{WTe}_{2}$.

The single crystal of NbP used for the present study and the respective X-ray diffraction patterns are shown in Fig. 2a. The crystal structure of $\mathrm{NbP}$ is non-centrosymmetric space group $I 4_{1} m d$ (Fig. 2b). No indication of twinning was found in the diffraction experiments. Both atom types have the same coordination number of six, and the same coordination environment in the form of a trigonal prism. A detailed overview of the structural characterization is presented in the Supplementary Information. A measurement of the temperature dependence of the resistivity, $\rho_{x x}(T)$, is a simple way to identify the electronic states of a material. On the basis of our high-quality single crystals of $\mathrm{NbP}$ grown via chemical vapour transport reactions, $\rho_{x x}(T)$ is measured under various transverse magnetic fields ranging from 0 to $9 \mathrm{~T}$, as shown in Fig. $2 \mathrm{c}$. At zero field, we observe metallic behaviour with $\rho_{x x}(300 \mathrm{~K})=73 \mu \Omega \mathrm{cm}$ and a residual resistivity $\rho_{x x}(2 \mathrm{~K})=0.63 \mu \Omega \mathrm{cm}$. This results in a residual resistivity ratio $\left[\rho_{x x}(300 \mathrm{~K}) / \rho_{x x}(2 \mathrm{~K})\right]=115$, which is directly related to the metallicity and quality of the crystal. Compared to other similar materials at low temperature $(2 \mathrm{~K}), \mathrm{NbP}$ exhibits a resistivity that is about 30 times lower than that of $\mathrm{WTe}_{2}$ (ref. 1) but 30 times higher than that of $\mathrm{Cd}_{3} \mathrm{As}_{2}$ (ref. 16). NbP does not become superconducting for temperatures above $0.10 \mathrm{~K}$. After applying a magnetic field, we observe a remarkable change in the resistivity. $\rho_{x x}(T)$ changes from a positive slope (metallic) to a negative slope (semiconducting) at a very small field of $0.1 \mathrm{~T}$, and becomes completely semiconducting at a field of $2 \mathrm{~T}$. This may be due to the opening of a gap at the Weyl point. In general, a conventional semimetal does not exhibit such behaviour, whereas some small-gap or gapless semimetals (for example, $\mathrm{WTe}_{2}$ (ref. 1) and $\mathrm{Cd}_{3} \mathrm{As}_{2}$ (ref. 16)) exhibit a similar trend, usually at very high fields and low temperature. Another important fact observed in the present material is that $\rho_{x x}(T)$ also increases markedly owing to the application of magnetic fields at room temperature $(300 \mathrm{~K})$.

We now focus on the MR measurement in NbP. The MR is commonly calculated as the ratio of the change in resistivity due to the applied magnetic field $(H),[(\rho(H)-\rho(0)) / \rho(0)] \times 100 \%$. Figure $2 \mathrm{~d}$ shows the MR measured in transverse magnetic fields up to $9 \mathrm{~T}$ at different temperatures. At low temperatures, we find that $\mathrm{NbP}$ exhibits an extremely large $\mathrm{MR}=8.5 \times 10^{5} \%$ at $1.85 \mathrm{~K}$ in a field of $9 \mathrm{~T}$. This MR is five times as large as that measured for the same field in $\mathrm{WTe}_{2}$ (ref. 1) and nearly twice as large as that of TaAs (ref. 34), another WSM predicted in the same family as NbP 

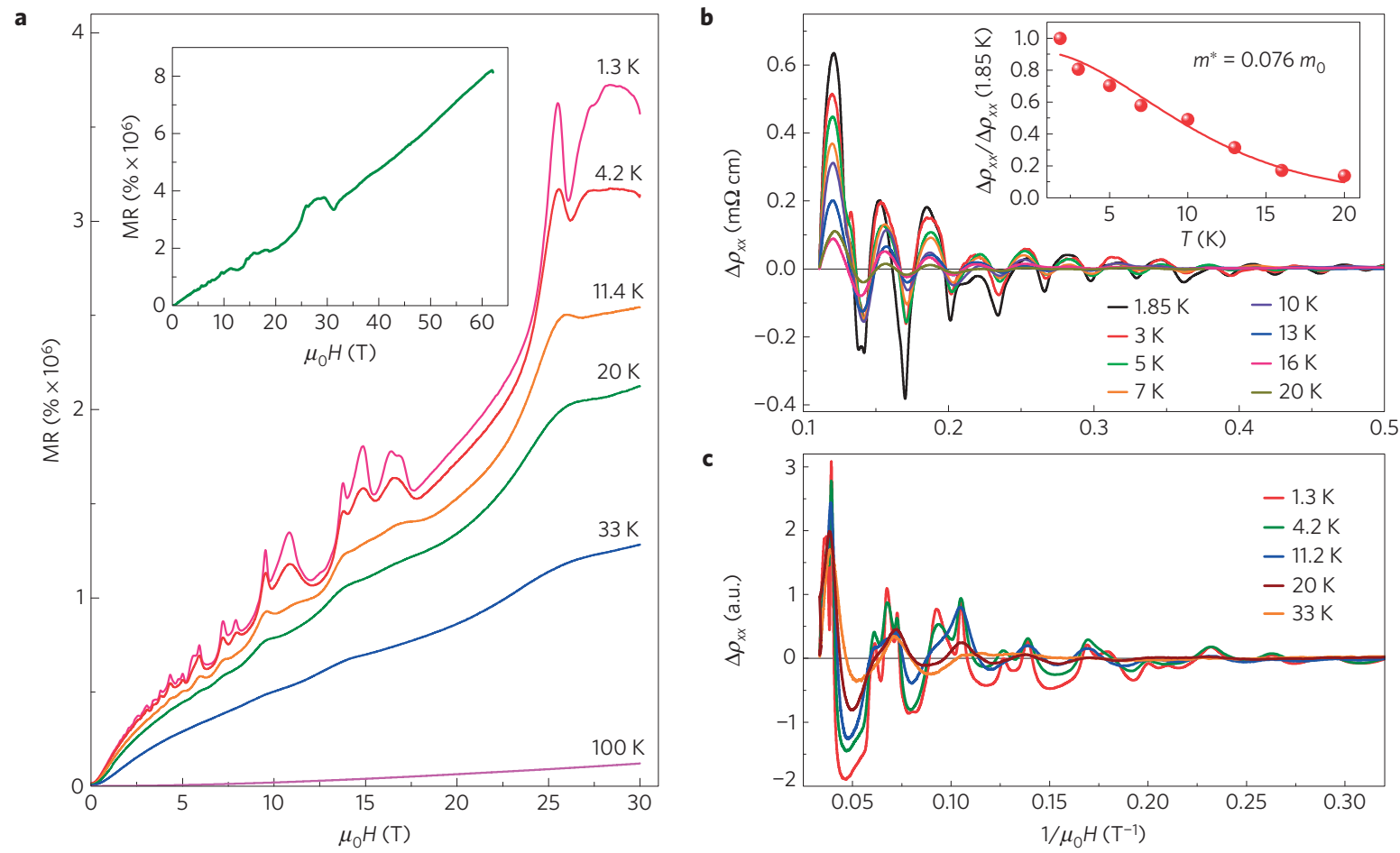

Figure 3 | High-field magnetoresistance and SdH oscillation. a, Transverse MR measured in static magnetic fields of up to $30 \mathrm{~T}$ at different temperatures. The inset of a shows the MR measured in a pulsed magnetic field up to $62 \mathrm{~T}$. b. SdH oscillations after subtracting the background from the $9 \mathrm{~T} \rho_{\mathrm{xx}}$ measurements. The inset of $\mathbf{b}$ shows the temperature dependence of the relative amplitude of $\Delta \rho_{x x}$ for the SdH oscillation at 8.2 T. The solid line is a fit to the Lifshitz-Kosevich formula. c, SdH oscillations after subtracting the background from the $30 \mathrm{~T} \rho_{x x}$ measurements.

(refs 10,11). On increasing the temperature, the MR of NbP remains almost unchanged up to $20 \mathrm{~K}$ and then starts to decrease at higher temperatures. We note that the MR is still as high as $250 \%$ in a field of $9 \mathrm{~T}$ at room temperature (inset of Fig. 2d). We have also measured the dependence of the MR on the direction of the magnetic field. Figure $2 \mathrm{f}$ shows the MR observed at different angles $(\theta)$ between the field direction and the current direction. The MR decreases slightly from $8.5 \times 10^{5} \%$ at $\theta=90^{\circ}$ (transverse) to $2.5 \times 10^{5} \%$ at $\theta=0^{\circ}$ (longitudinal). Thus, the MR varies by a factor of only 3.4, implying a relatively isotropic nature of the material compared with the layered semimetal $\mathrm{WTe}_{2}$.

A large MR is usually associated with a high mobility. The carrier mobility and concentration are two important parameters of a material that can be derived from the Hall coefficient. We have performed Hall effect measurements in both temperaturesweep and field-sweep modes to improve the accuracy of our data. The field dependence of the Hall resistivity $\rho_{x y}(H)$ exhibits a linear characteristic at high fields (see Supplementary Information). However, the nonlinear behaviour in low fields indicates the involvement of more than one type of charge carrier in the transport properties. As seen from the inset of Fig. 2e, NbP exhibits a negative Hall coefficient, $R_{\mathrm{H}}(T)$, up to $125 \mathrm{~K}$, which changes sign for temperatures above $125 \mathrm{~K}$. For the sake of simplicity, we use the single-carrier Drude band model, $n_{\mathrm{e}, \mathrm{h}}(T)=1 /\left[e R_{\mathrm{H}}(T)\right]$, to calculate the carrier density and $\mu_{\mathrm{e}, \mathrm{h}}(T)=R_{\mathrm{H}}(T) / \rho_{x x}(T)$ to estimate the mobility, where $n_{\mathrm{e}}\left(n_{\mathrm{h}}\right)$ and $\mu_{\mathrm{e}}\left(\mu_{\mathrm{h}}\right)$ are the charge density and mobility of the electron (hole), respectively. We use the slope of $\rho_{x y}(H)$ at high fields to calculate the Hall coefficient (Fig. 2e). The electron carrier concentration, $n_{\mathrm{e}}$, is found to be $1.5 \times 10^{18} \mathrm{~cm}^{-3}$ at $1.85 \mathrm{~K}$, and increases slowly with temperature, exhibiting a semimetal-like or very small gap-like behaviour. The mobility plays a major role in the charge transport in a material and consequently determines the efficiency of various devices. Here, NbP exhibits an ultrahigh mobility of $5 \times 10^{6} \mathrm{~cm}^{2} \mathrm{~V}^{-1} \mathrm{~s}^{-1}$ at $1.85 \mathrm{~K}$. This value is close to that of $\mathrm{Cd}_{3} \mathrm{As}_{2}\left(9 \times 10^{6} \mathrm{~cm}^{2} \mathrm{~V}^{-1} \mathrm{~s}^{-1}\right.$; ref. 16), one order magnitude higher than that of TaAs (ref. 34) at $2 \mathrm{~K}$. We note that the current mobility is extracted from a simple one-band model that neglects anisotropy. This averaged mobility is below the record mobility measured in $\mathrm{Bi}\left(10^{8} \mathrm{~cm}^{2} \mathrm{~V}^{-1} \mathrm{~s}^{-1}\right.$; ref. 37$)$. Furthermore, it has been shown that the mobility in $\mathrm{Cd}_{3} \mathrm{As}_{2}$ scales with the residual resistivity. Hence, it can be proposed that materials with a low residual resistivity exhibit high mobilities, with the present case being a good example.

The low-field measurements (Fig. 2d,f) show no saturation of the $\mathrm{MR}$ up to $9 \mathrm{~T}$ in the entire temperature range. To pursue this finding to even higher fields, we have performed transverse MR measurements up to $30 \mathrm{~T}$ in d.c. magnetic fields, as shown in Fig. $3 \mathrm{a}$. The MR increases to $3.6 \times 10^{6} \%$ for a field of $30 \mathrm{~T}$ at $1.3 \mathrm{~K}$, and still shows no tendency of saturation. We have further corroborated this trend of a non-saturating magnetoresistance by performing experiments in pulsed magnetic fields up to $62 \mathrm{~T}$ at $1.5 \mathrm{~K}$ (inset of Fig. 3a). The MR continues to increase with magnetic field up to $62 \mathrm{~T}$, the maximum field reached in the pulsed magnetic field experiments, when we find a MR of $8.1 \times 10^{4}$ (or $8.1 \times 10^{6} \%$ ). In a field as high as $60 \mathrm{~T}$, we note that $\mathrm{WTe}_{2}$ (ref. 1) and $\mathrm{Bi}$ (ref. 25) are reported to exhibit a MR of $1.3 \times 10^{5}$ and $1 \times 10^{6}$, respectively. As already indicated in the $9 \mathrm{~T}$ MR data depicted in Fig. 2d,f, SdH quantum oscillations appear for $T \leq 30 \mathrm{~K}$. At the lowest temperature $(1.85 \mathrm{~K})$ the oscillations start for fields as low as $1 \mathrm{~T}$. Because SdH oscillations appear only when the energy spacing between two Landau levels is larger than their broadening due to disorder, we can estimate a lower limit to the quantum mobility of the carriers involved, $\mu_{\mathrm{q}}>10^{5} \mathrm{~cm}^{2} \mathrm{~V}^{-1} \mathrm{~s}^{-1}$, in agreement with the large electron (transport) mobility extracted from the Hall effect.

The physical parameters of the charge carriers are derived from the Fermi surface that is measured by the oscillations observed in the transport properties. Both $\rho_{x x}$ and $\rho_{x y}$ measured up to $9 \mathrm{~T}$ exhibit very clear $\mathrm{SdH}$ oscillations starting from $1 \mathrm{~T}$. This 

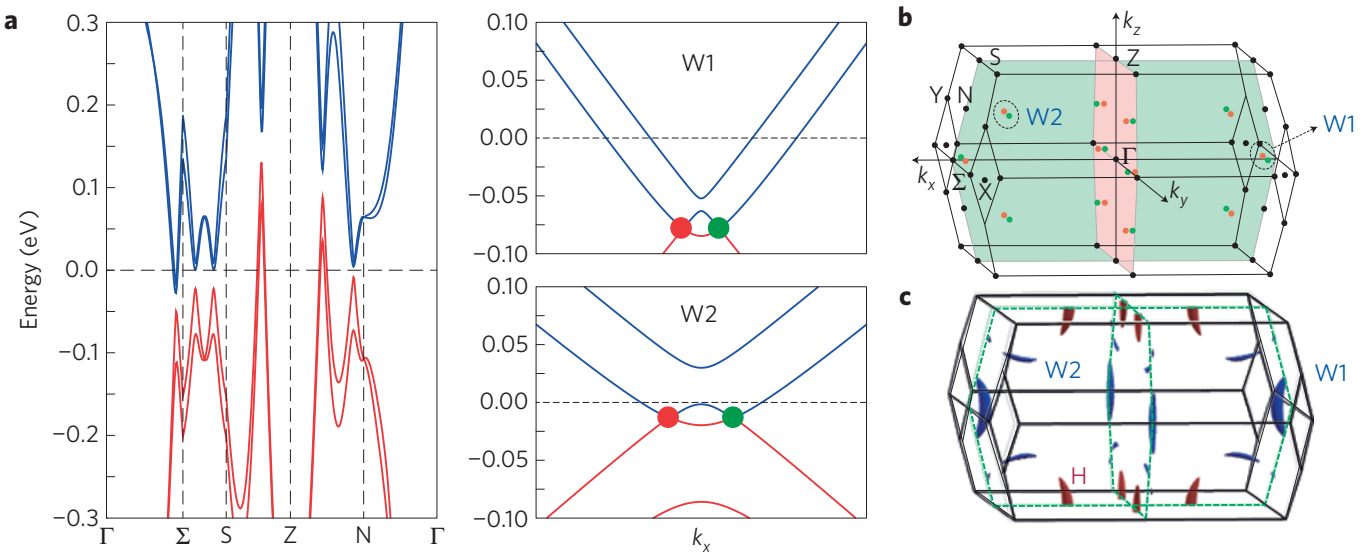

Figure 4 | Bulk band structures of NbP. a, Ab initio band structure. The dispersions along $k_{y}$ connecting a pair of Weyl points with opposite chirality are plotted for the W1- and W2-types of Weyl points in the right panels. Red and green circles represent positive and negative chiral Weyl points, respectively. The Fermi energy is shifted to zero. The valence and conduction bands are indicated by the red and blue lines, respectively. $\mathbf{b}$, The first Brillouin zone in momentum space for the primitive unit cell. All twelve pairs of Weyl points are illustrated, in which W1 and W2-types of Weyl points are indicated. c, Bulk Fermi surfaces. The blue and red surfaces represent electron (W1 and W2) and hole (H) pockets, respectively. The Fermi energy is chosen as the slightly electron-doped case to qualitatively match the experiment.

indicates a very low effective mass, resulting in a high mobility. To obtain the amplitude of the SdH oscillations, $\Delta \rho_{x x}$, from $\rho_{x x}$, we subtracted a smooth background. The results are plotted as a function of $1 /\left(\mu_{0} H\right)$ at various temperatures in Fig. $3 \mathrm{~b}$. As expected, the oscillations are periodic in $1 /\left(\mu_{0} H\right)$. They occur owing to the quantization of energy levels, which directly gives the effective mass of the charge carriers. We observe three different oscillation frequencies of $F=7 \mathrm{~T}, 13 \mathrm{~T}$ and $32 \mathrm{~T}$ from the data shown in Fig. $3 b$, which is consistent with the existence of tiny carrier pockets near the Weyl points in the calculated Fermi surface (Fig. 4). We note that these frequencies depend sensitively on the field direction. For example, the frequency of $F=32 \mathrm{~T}$, which is equivalent to the periodicity $1 /\left(\mu_{0} \Delta H\right)=0.03127 \mathrm{~T}^{-1}$, corresponds to a cross-sectional area of the Fermi surface $A_{\mathrm{F}}=0.003 \AA^{-2}$ from the Onsager relationship $F=\left(\Phi_{0} / 2 \pi^{2}\right) A_{\mathrm{F}}$, where $\Phi_{0}$ is the magnetic flux quantum. This is a tiny area, only $0.08 \%$ of the cross-sectional area of the first Brillouin zone. Supposing a circular cross-section, a very small Fermi momentum $k_{\mathrm{F}}=0.0312 \AA^{-1}$ is obtained. The cyclotron effective mass of the carriers is determined by fitting the temperature dependence of amplitude of the oscillations to the Lifshitz-Kosevich formula,

$$
\frac{\Delta \rho_{x x}(T, B)}{\rho_{x x}(0)}=\mathrm{e}^{-2 \pi^{2} k_{\mathrm{B}} T_{\mathrm{D}} / \beta} \frac{2 \pi^{2} k_{\mathrm{B}} T / \beta}{\sinh \left(2 \pi^{2} k_{\mathrm{B}} T / \beta\right)}
$$

where $k_{\mathrm{B}}$ is Boltzmann's constant, $\beta=e h B / 2 \pi m^{*}$ and $T_{\mathrm{D}}=h / 4 \pi^{2} \tau k_{\mathrm{B}}$ are the fitting parameters, which directly results in the effective mass $m^{*}$ and quantum lifetime $\tau$ of the charge carriers. The value of $m^{*}$ calculated from the temperature-dependent $\mathrm{SdH}$ oscillations in a field of $8.2 \mathrm{~T}$ is $0.076 m_{0}$, where $m_{0}$ is the bare mass of the electron. This value is comparable to that reported for $\mathrm{Cd}_{3} \mathrm{As}_{2}$ (ref. 16). We also find a very large Fermi velocity $v_{\mathrm{F}}=\hbar k_{\mathrm{F}} / \mathrm{m}^{*}=4.8 \times 10^{5} \mathrm{~m} \mathrm{~s}^{-1}$. The large Fermi velocity and low effective mass are responsible for the observed ultrahigh mobility in NbP.

The SdH oscillations at different temperatures obtained from the $30 \mathrm{~T}$ dc magnetic field measurements are plotted in Fig. 3c. At the lowest temperature, marked by arrows, the $\mathrm{SdH}$ maxima at $8 \mathrm{~T}$ $\left(0.125 \mathrm{~T}^{-1}\right)$ and $11 \mathrm{~T}\left(0.091 \mathrm{~T}^{-1}\right)$ start to split into two distinct peaks, and the maximum at $16 \mathrm{~T}\left(0.0625 \mathrm{~T}^{-1}\right)$ develops into four peaks. This can be assigned to the lifting of the spin degeneracy and the degeneracy of the dual Weyl point.

To further understand the transport properties and verify the Weyl physics of NbP, we have performed $a b$ initio band-structure calculations. NbP crystallizes in a body-centred-tetragonal lattice with the non-symmorphic space group $I 4_{1} m d$. The lack of inversion symmetry of the lattice leads to the lifting of spin degeneracy in the band structure. Near the Fermi energy, twelve pairs of Weyl points lie aside the central planes in the Brillouin zone, consistent with recent calculations ${ }^{10,11}$. Twelve pairs of Weyl points can be classified into two groups, labelled as W1 and W2 (Fig. 4b). The W1 pair that lies in the $k_{z}=0$ plane is lower in energy that the W2 pair off the $k_{z}=0$ plane. An important feature in the band structure is that the Fermi energy crosses the quadratic-type valence bands and also the linear Weyl-type conduction bands, leading to very small hole and electron pockets on the Fermi surface, respectively. Four equivalent hole pockets appear around the $\mathrm{Z}$ point (labelled as $\mathrm{H}$ in Fig. 4c). Four larger and eight smaller electron pockets exist near the $\Sigma$ and $\mathrm{N}$ points, respectively, corresponding to W1 and W2 Weyl bands. This is consistent with multiple tiny Fermi surface areas with low effective masses extracted from SdH oscillations. We note that the specific shape of the Fermi surfaces relies sensitively on the position of the Fermi energy, which is determined by the dopant concentration of the sample. In Fig. $4 c$ we set the Fermi energy as the ideal electron-hole compensation case. Furthermore, from the bulk to the surface, as a manifestation of topology, Fermi arcs exist between the projection of Weyl points with opposite chirality. Angle-resolved photoemission spectroscopy (ARPES) experiments are called for to verify $\mathrm{NbP}$ as a WSM by investigating the surface states.

As we have demonstrated here, $\mathrm{NbP}$ is an exotic semimetal with interesting transport properties. As seen in the band structure, it combines the electronic structures of a normal semimetal and a WSM together. Similar to normal semimetals such as $\mathrm{WTe}_{2}$ and $\mathrm{Bi}$, $\mathrm{NbP}$ exhibits both electron and hole pockets at different positions in the Brillouin zone; however, unlike $\mathrm{WTe}_{2}$ and $\mathrm{Bi}$, its electron pockets are relevant to the linear Weyl bands, from which the high mobility of the high-quality samples may originate. In contrast to Weyl semimetals (for example, TaAs) and Dirac semimetals (for example, $\mathrm{Cd}_{3} \mathrm{As}_{2}$ ), in which the Fermi energy may cross only one type of band (electron or hole), NbP naturally hosts both types of carriers and consequently exhibits a huge MR in an electron-hole resonance situation.

\section{Methods}

Methods and any associated references are available in the online version of the paper. 
Received 16 March 2015; accepted 19 May 2015; published online 22 June 2015

\section{References}

1. Ali, M. N. et al. Large, non-saturating magnetoresistance in $\mathrm{WTe}_{2}$. Nature 514, 205-208 (2014)

2. Wan, X. G., Turner, A. M., Vishwanath, A. \& Savrasov, S. Y. Topological semimetal and Fermi-arc surface states in the electronic structure of pyrochlore iridates. Phys. Rev. B 83, 205101 (2011).

3. Novoselov, K. et al. Two-dimensional gas of massless Dirac fermions in graphene. Nature 438, 197-200 (2005).

4. Turner, A. M. \& Vishwanath, A. Beyond band insulators: Topology of semi-metals and interacting phases. Preprint at http://arxiv.org/abs/1301.0330 (2013).

5. Hosur, P. \& Qi, X. L. Recent developments in transport phenomena in Weyl semimetals. C. R. Phys. 14, 857-870 (2013).

6. Vafek, O. \& Vishwanath, A. Dirac Fermions in solids: From high- $T_{c}$ cuprates and graphene to topological insulators and Weyl semimetals. Annu. Rev. Condens. Matter Phys. 5, 83-112 (2014).

7. Parameswaran, S. A., Grover, T., Abanin, D. A., Pesin, D. A. \& Vishwanath, A. Probing the chiral anomaly with nonlocal transport in three-dimensional topological semimetals. Phys. Rev. X 4, 031035 (2014)

8. Qi, X-L. \& Zhang, S-C. Topological insulators and superconductors. Rev. Mod. Phys. 83, 1057-1110 (2011).

9. Hasan, M. Z. \& Kane, C. L. Colloquium: Topological insulators. Rev. Mod. Phys. 82, 3045-3067 (2010)

10. Weng, H., Fang, C., Fang, Z., Bernevig, B. A. \& Dai, X. Weyl semimetal phase in noncentrosymmetric transition-metal monophosphides. Phys. Rev. X 5, 011029 (2015).

11. Huang, S-M. et al. An inversion breaking Weyl semimetal state in the TaAs material class. Preprint at http://arxiv.org/abs/1501.00755 (2015).

12. Burkov, A. A. \& Balents, L. Weyl semimetal in a topological insulator multilayer. Phys. Rev. Lett. 107, 127205 (2011).

13. Xu, G., Weng, H., Wang, Z., Dai, X. \& Fang, Z. Chern semimetal and the quantized anomalous Hall effect in $\mathrm{HgCr}_{2} \mathrm{Se}_{4}$. Phys. Rev. Lett. 107, 186806 (2011)

14. Wang, Z. et al. Dirac semimetal and topological phase transitions in $A_{3} \mathrm{Bi}$ ( $A=\mathrm{Na}, \mathrm{K}, \mathrm{Rb}$ ). Phys. Rev. B 85, 195320 (2012).

15. Wang, Z., Weng, H., Wu, Q., Dai, X. \& Fang, Z. Three-dimensional Dirac semimetal and quantum transport in $\mathrm{Cd}_{3} \mathrm{As}_{2}$. Phys. Rev. B 88, 125427 (2013).

16. Liang, T. et al. Ultrahigh mobility and giant magnetoresistance in the Dirac semimetal $\mathrm{Cd}_{3} \mathrm{As}_{2}$. Nature Mater. 14, 280-284 (2014).

17. Narayanan, A. et al. Linear magnetoresistance caused by mobility fluctuations in n-doped $\mathrm{Cd}_{3} \mathrm{As}_{2}$. Phys. Rev. Lett. 114, 117201 (2015).

18. Baibich, M. N., Broto, J. M., Fert, A., Van Dau, F. N. \& Petroff, F. Giant magnetoresistance of (001)Fe/(001)Cr magnetic superlattices. Phys. Rev. Lett. 61, 2472-2475 (1988)

19. Binasch, G., Grünberg, P., Saurenbach, F. \& Zinn, W. Enhanced magnetoresistance in layered magnetic structures with antiferromagnetic interlayer exchange. Phys. Rev. B 39, 4828-4830 (1989).

20. Parkin, S. et al. Magnetically engineered spintronic sensors and memory. Proc. IEEE 91, 661-680 (2003).

21. Singleton, J. Band Theory and Electronic Properties of Solids (Oxford Univ. Press, 2001).

22. Mangez, J. H., Issi, J. P. \& Heremans, J. Transport properties of bismuth in quantizing magnetic fields. Phys. Rev. B 14, 4381-4385 (1976).
23. Heremans, J. et al. Cyclotron resonance in epitaxial $\mathrm{Bi}_{1-x} \mathrm{Sb}_{x}$ films grown by molecular-beam epitaxy. Phys. Rev. B 48, 11329-11335 (1993).

24. Yang, F. Y. et al. Large magnetoresistance of electrodeposited single-crystal bismuth thin films. Science 284, 1335-1337 (1999).

25. Fauqué, B., Vignolle, B., Proust, C., Issi, J-P. \& Behnia, K. Electronic instability in bismuth far beyond the quantum limit. New J. Phys. 11, 113012 (2009).

26. $\mathrm{Xu}, \mathrm{R}$. et al. Large magnetoresistance in non-magnetic silver chalcogenides. Nature 390, 57-60 (1997).

27. Zhang, W. et al. Topological aspect and quantum magnetoresistance of $\beta-\mathrm{Ag}_{2}$ Te. Phys. Rev. Lett. 106, 156808 (2011).

28. Chadov, S. et al. Tunable multifunctional topological insulators in ternary Heusler compounds. Nature Mater. 9, 541-545 (2010).

29. Shekhar, C. et al. Ultrahigh mobility and nonsaturating magnetoresistance in Heusler topological insulators. Phys. Rev. B 86, 155314 (2012).

30. Yan, B. \& de Visser, A. Half-Heusler topological insulators. MRS Bull. 39, 859-866 (2014)

31. He, L. P. et al. Quantum transport evidence for the three-dimensional Dirac semimetal phase in $\mathrm{Cd}_{3} \mathrm{As}_{2}$. Phys. Rev. Lett. 113, 246402 (2014).

32. Feng, J. et al. Large linear magnetoresistance in Dirac semi-metal $\mathrm{Cd}_{3} \mathrm{As}_{2}$ with Fermi surfaces close to the Dirac points. Preprint at http://arxiv.org/abs/1405.6611v1 (2014).

33. He, L. P. et al. Quantum transport evidence for the three-dimensional Dirac semimetal phase in $\mathrm{Cd}_{3} \mathrm{As}_{2}$. Phys. Rev. Lett. 113, 246402 (2014).

34. Zhang, C. et al. Tantalum monoarsenide: An exotic compensated semimetal. Preprint at http://arxiv.org/abs/1502.00251 (2015).

35. Parish, M. M. \& Littlewood, P. B. Non-saturating magnetoresistance in heavily disordered semiconductors. Nature 426, 162-165 (2003).

36. Abrikosov, A. Quantum magnetoresistance. Phys. Rev. B 58, 2788-2794 (1998).

37. Collaudin, A., Fauqué, B., Fuseya, Y., Kang, W. \& Behnia, K. Angle dependence of the orbital magnetoresistance in bismuth. Phys. Rev. X 5, 021022 (2015).

\section{Acknowledgements}

This work was financially supported by the Deutsche Forschungsgemeinschaft DFG (Project No.EB 518/1-1 of DFG-SPP 1666 'Topological Insulators') and by the ERC Advanced Grant No. (291472) 'Idea Heusler'. Y.C. acknowledge support from the EPSRC (UK) grant EP/K04074X/1 and a DARPA (US) MESO project (no. N66001-11-1-4105). We acknowledge the support of the High Magnetic Field Laboratory Dresden (HLD) at HZDR and High Field Magnet Laboratory Nijmegen (HFML-RU/FOM), members of the European Magnetic Field Laboratory (EMFL).

\section{Author contributions}

B.Y. conceived the original idea for the project. C.S. performed the low-field PPMS measurement with the help of M.N. and W.S. C.S., I.L. and U.Z. performed the $30 \mathrm{~T}$ static magnetic field measurements. Y.Skourski, A.K.N. and J.W. performed the pulsed high magnetic field experiments. M.S. grew the single-crystal samples. Y.Sun and B.Y. calculated band structures. H.B. and Y.G. characterized the crystal structure. Z.L. and Y.C. contributed to helpful discussions. All authors analysed the results. B.Y., C.S. and A.K.N. wrote the manuscript with substantial contributions from all authors. C.F. supervised the project.

\section{Additional information}

Supplementary information is available in the online version of the paper. Reprints and permissions information is available online at www.nature.com/reprints.

Correspondence and requests for materials should be addressed to B.Y.

\section{Competing financial interests}

The authors declare no competing financial interests. 


\section{Methods}

High-quality single crystals of $\mathrm{NbP}$ were grown via a chemical vapour transport reaction using iodine as a transport agent. Initially, a polycrystalline powder of NbP was synthesized by a direct reaction of niobium (Chempur 99.9\%) and red phosphorus (Heraeus 99.999\%) kept in an evacuated fused silica tube for $48 \mathrm{~h}$ at $800^{\circ} \mathrm{C}$. Starting from this microcrystalline powder, the single crystals of $\mathrm{NbP}$ were synthesized by chemical vapour transport in a temperature gradient starting from $850{ }^{\circ} \mathrm{C}$ (source) to $950^{\circ} \mathrm{C}$ (sink) and a transport agent with a concentration of $13.5 \mathrm{mg} \mathrm{cm}^{-3}$ iodine (Alfa Aesar 99.998\%; ref. 38). The orientation and crystal structure of the present single crystal were investigated using the diffraction data sets collected on a Rigaku AFC7 diffractometer equipped with a Saturn 724+ charge-coupled device detector (monochromatic Mo K $\alpha$ radiation, $\lambda=0.71073 \AA$ ). Structure refinement was performed by full-matrix least-squares on $F$ using the program package WinCSD.

The transport measurements were performed in various physical property measurement systems (PPMS, Quantum Design, ACT option, home build adiabatic demagnetization stage). The $30 \mathrm{~T}$ static magnetic field measurements were performed at the High Field Magnet Laboratory HFML-RU/FOM in Nijmegen, and the pulsed magnetic field experiments were carried out at the
Dresden High Magnetic Field Laboratory HLD-HZDR; both laboratories are members of the European Magnetic Field Laboratory (EMFL).

The $a b$ initio calculations were performed within the framework of density functional theory (DFT), implemented in the Vienna $a b$ initio simulation package $^{39}$. The core electrons were represented by the projector-augmented-wave potential and generalized gradient approximation (GGA) are employed for the exchange correlation functional. We interpolated the bulk Fermi surface using maximally localized Wannier functions (MLWFs; ref. 40).

\section{References}

38. Martin, J. \& Gruehn, R. Zum chemischen Transport von Monophosphiden MP $(M=\mathrm{Zr}$, Hf, Nb, Ta, Mo, W) und Diposphiden $M \mathrm{P} 2(M=\mathrm{Ti}, \mathrm{Zr}, \mathrm{Hf})$. Z. Kristallogr. 182, 180-182 (1988).

39. Kresse, G. \& Furthmüller, J. Efficient iterative schemes for $a b$ initio total-energy calculations using a plane-wave basis set. Phys. Rev. B 54, 11169-11186 (1996).

40. Mostofi, A. A. et al. wannier90: A tool for obtaining maximally-localised Wannier functions. Comput. Phys. Commun. 178, 685-699 (2008). 